\title{
Non-invasive three-dimensional localisation of arrhythmogenic foci in Wolff-Parkinson-White syndrome and in ventricular tachycardia by radionuclide ventriculography: phase analysis of double-angulated integrated single photon emission computed tomography (SPECT)
}

Department of Cardiology, Angiology, and Pneumology, University of Ulm, Germany $\mathrm{P}$ Weismüller P Richter

$M$ Kochs

V Hombach

Department of

Nuclear Medicine,

University of Ulm,

Federal Republic of

Germany

$M$ Clausen

$\mathrm{R}$ Weller

J Steinmann

E Henze

W E Adam

AEC-Institute of Life Science, Faculty of Medicine, Universiteit van Pretoria, South Africa

I Dormehl

Correspondence to: Dr Peter Weismüller, Department of Cardiology, Angiology and Pneumology, University of Ulm, 8 Robert-Koch Street, 7900 Ulm, Germany.

Accepted for publication 15 September 1992

P Weismüller, M Clausen, R Weller, P Richter, J Steinmann, E Henze, I Dormehl, $M$ Kochs, W E Adam, V Hombach

\begin{abstract}
A new tomographic technique combined with phase analysis was used to detect premature and ectopic ventricular contraction patterns in 15 patients with Wolff-Parkinson-White syndrome and during ventricular tachycardia in seven patients. Data generated by gated singlephoton emission computed tomography (SPECT) were analysed by backprojection of the Fourier coefficients, doubleangulation, and integration to thick slices containing the ventricles, thus allowing visualisation of the contraction patterns in three perpendicular views. The results were compared with those of catheter mapping.
\end{abstract}

In nine patients with Wolff-ParkinsonWhite syndrome the site of initial contraction detected was identical with the site of the accessory pathway found by catheter mapping. The sites of origin of the ventricular tachycardias determined by catheter mapping were within $3 \mathrm{~cm}$ of the sites detected by the new technique.

This new technique seems to be a promising non-invasive method for localising ectopic ventricular activity that will considerably shorten the time required for subsequent invasive procedures.

\section{(Br Heart f 1993;69:201-210)}

The exact site of the accessory pathway must be identified before patients with WolffParkinson-White syndrome (WPW syndrome) can be treated with catheter ablation. Similarly, the detection of the site of origin of tachycardia is essential for intraoperative ${ }^{1}$ or catheter ablation ${ }^{2}$ in patients with sustained ventricular tachycardia. Invasive catheter mapping is the standard method used to localise accessory pathways in WPW syndrome $^{3}$ and identify the site of origin of ventricular tachycardias ${ }^{4}$ before interventional procedures.

It is difficult to localise anterograde con- ducting accessory pathways from the surface electrocardiogram. ${ }^{56}$ Also the determination of the origin of ventricular tachycardias from the configuration of the surface electrocardiogram is inaccurate. ${ }^{78} \mathrm{We}$ and others have shown that phase analysis of radionuclide ventriculography is able to show the local contraction pattern and thus the site of initial

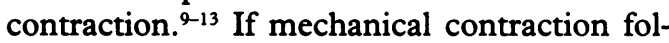
lows the electrical excitation, localisation of ectopic ventricular depolarisation is possible. When phase analysis of planar radionuclide ventriculography was used to localise ectopic ventricular excitation in WPW patients ${ }^{14-16}$ and in patients with sustained ventricular tachycardia ${ }^{17-19}$ the results corresponded well with the results of invasive mapping. In contrast, experimental data showed that this method is only able to detect an extended area of initial contraction ${ }^{20}$ because the ventricles are displayed separately in only one projection (left anterior oblique). Even in this projection other cardiac structures are superimposed on the ventricles. In a previous study we reported the preliminary results of singlephoton emission computed tomography (SPECT) and integrated slices that provide three dimensional cross sectional images of the heart derived from phase analysis. ${ }^{21} 22$ In addition to the left anterior oblique view of conventional planar phase analysis, an axial view of the heart was generated, which was perpendicular to the long axis of the body.

This study reports on the first clinical data of phase analysis of radionuclide ventriculography obtained with a newly developed computer algorithm for analysing gated SPECT data. The method allows images to be generated in any projection of the cardiac blood pool with isolated display of the ventricular chambers.

\section{Patients and methods}

CONTROL GROUP

Forty patients with normal atrioventricular conduction in the surface electrocardiogram 
made up the control group. Doubleangulated integrated SPECT was performed during sinus rhythm to assess left ventricular function.

PATIENTS WITH WPW SYNDROME

We studied four women and 11 men $(41.9$ (13.9) 25-71 years). All of them had a delta wave in the surface electrocardiogram. Seven of them were studied during preexcitation of the ventricles caused by depolarisation through the accessory pathway and also during normal atrioventricular conduction with a narrow QRS complex: six patients after administration of ajmaline $(50 \mathrm{mg}$ intravenously) and one after successful catheter ablation.

In 14 patients catheter mapping was performed during an electrophysiological study and the atrial insertion of the accessory pathway was determined by the following criteria: shortest $\mathrm{P}$-delta interval measured during stimulation with the mapping electrode, shortest VA interval during atrioventricular reentrant tachycardia and shortest VA interval during ventricular pacing. In all patients the whole atrioventricular ring was scanned by the mapping electrode to detect multiple bypass tracts. In one of these patients, catheter mapping could not be performed during the first mapping procedure because repeated atrial fibrillation episodes were induced mechanically by the mapping catheter. In two patients (case 19 and case 16) successful radiofrequency catheter ablation of the atrial bypass insertion was performed. ${ }^{23}$

\section{PATIENTS WITH VENTRICULAR TACHYCARDIA}

Only patients with haemodynamically well tolerated ventricular tachycardias were investigated. The mean (SD) age of the patients was $56.3(12.2)$ years (range 32-70). Six had coronary artery disease and one had right ventricular dysplasia with areas of fatty degeneration in the left ventricle and in the septum detected by magnetic resonance imaging. In all but one patient two radionuclide studies were performed-during clinical ventricular tachycardia and then during sinus rhythm. Table 1 shows the details of these patients. The mean ejection fraction measured from left ventricular angiography was $39 \cdot 3(14 \cdot 8) \%(24-68 \%)$. The mean ejection fraction during tachycardia was $27 \cdot 3(7 \cdot 7) \%$ $(17-38 \%)$ as determined by planar radionuclide ventriculography. The mean rate of the ventricular tachycardias was 152 (19) min $(120-175 / \mathrm{min})$. In all patients the origin of the tachycardia could be detected invasively, $\frac{7}{c}$ either by catheter mapping ${ }^{4}$ or by intraoperative mapping (case 4). All patients presented $\underset{\vec{S}}{\overrightarrow{7}}$ with one clinical ventricular tachycardia. Two $\bar{c}$ patients showed another configuration of a 흘 non-clinical ventricular tachycardia. The site $\frac{\bar{\omega}}{\vec{D}}$ of origin of the clinical tachycardia was deter- $\propto$ mined by the detection of a mid diastolic is potential and by pace-mapping. ${ }^{4} \mathrm{~A}$ diastolic $\vec{\circ}$ potential during tachycardia was found in six patients, and preceded the QRS complex by $\vec{\omega}$ $60 \mathrm{~ms}(15-100 \mathrm{~ms})$ on average. The criterion of pace mapping was fulfilled when the QRS complex paced by the mapping catheter was 0 identical in more than 10 of the 12 surface leads with the configuration of clinical tachycardia on the electrocardiogram. This occurred in three of the patients. In three patients (cases 2,3 , and 7 ) catheter ablation was per- 3 formed, in two of them (cases 3 and 7) by radiofrequency catheter ablation ${ }^{24}$ and in the other (case 2) by direct current. ${ }^{25}$ In one $\vec{\oplus}$ patient (case 4) the tachycardia could be tolerated haemodynamically for $20 \mathrm{~min}$ only after long-term administration of amiodarone. In this patient the site of origin of the clinical tachycardia was determined intraoperatively during surgical ablation.

\section{MODE OF INDUCTION OF THE VENTRICULAR TACHYCARDIA}

In all patients with ventricular tachycardia the tachycardia was induced directly after a basic $\overrightarrow{\overparen{D}}$ or control electrophysiological study and the stimulation was delivered through a catheter placed in the apex of the right ventricle. Single or double premature stimuli were used.

\section{DOUBLE-ANGULATED INTEGRATED SPECT}

(DA-ISPECT)

After in vivo labelling of the patients' ery- N throcytes with $740 \mathrm{MBq}(20 \mathrm{mCi})$ tech-

Table 1 Details of patients with ventricular tachycardia

\begin{tabular}{|c|c|c|c|c|c|c|c|c|c|}
\hline $\begin{array}{l}\text { Case } \\
\text { No }\end{array}$ & Sex & $\begin{array}{l}\text { Age } \\
(y r)\end{array}$ & Disease & $\begin{array}{l}E F(S R) \\
(\%)\end{array}$ & $\begin{array}{l}E F(V T) \\
(\%)\end{array}$ & $\begin{array}{l}V T \text { rate } \\
\text { (/min) }\end{array}$ & $\begin{array}{l}V T \\
\text { configuration }\end{array}$ & $\begin{array}{l}\text { ORS } \\
\text { (ms) }\end{array}$ & $\begin{array}{l}\text { Axis } \\
()\end{array}$ \\
\hline 1 & $M$ & 55 & $\begin{array}{l}\text { CAD } \\
\text { PW-An }\end{array}$ & 37 & 29 & 150 & LBBB & 180 & +120 \\
\hline 2 & $\mathbf{M}$ & 70 & $\begin{array}{l}\text { CAD } \\
\text { AW-An } \\
\text { PW-An }\end{array}$ & 36 & 21 & 171 & LBBB & 180 & +110 \\
\hline $\begin{array}{l}3 \\
4\end{array}$ & $\begin{array}{l}\mathrm{M} \\
\mathrm{M}\end{array}$ & $\begin{array}{l}32 \\
56\end{array}$ & $\begin{array}{l}\text { RVD } \\
\text { CAD } \\
\text { AW-An }\end{array}$ & $\begin{array}{l}68 \\
24\end{array}$ & $\frac{38}{-}$ & $\begin{array}{l}150 \\
158\end{array}$ & $\begin{array}{l}\text { RBBB } \\
\text { LBBB }\end{array}$ & $\begin{array}{l}130 \\
180\end{array}$ & $\begin{array}{r}-90 \\
0\end{array}$ \\
\hline 5 & $\mathrm{~F}$ & 58 & $\begin{array}{l}\text { CAD } \\
\text { AW-An }\end{array}$ & 37 & 26 & 120 & $\mathrm{RBBB}$ & 180 & -60 \\
\hline 6 & $\mathbf{M}$ & 67 & $\begin{array}{l}\text { CAD } \\
\text { PW-An }\end{array}$ & 47 & 33 & 175 & RBBB & 130 & -60 \\
\hline 7 & $\mathbf{M}$ & 56 & $\begin{array}{l}\text { CAD } \\
\text { AW-An }\end{array}$ & 26 & 17 & 142 & RBBB & 170 & +100 \\
\hline
\end{tabular}

The mean (2SD) ejection fraction measured by angiography during sinus rhythm is $39 \cdot 3(14 \cdot 8) \%$. Mean ejection fraction during The mean (2SD) ejection fraction measured by angiography during sinus rhy thm is a radionuclide ventriculography could not be tachycardia (planar radionuclide ventriculography) is $27 \cdot 3(7 \cdot 7) \%$. In patient 4 planar radionuclide ventriculographe used to determine the ejection fraction during ventricular tachycardia because of poor haemodynamic tolerance. plasia. 
netium $99 \mathrm{~m}$ pertechnetate we performed ECG-triggered $180^{\circ}$ SPECT with a 64 matrix, 32 angles, $15 \mathrm{~s}$ acquisition per step and 8 frames per cycle (single-headed camera: Orbiter, Siemens). Fourteen minutes of active data acquisition was needed and six minutes for the deposition of the data. The parameters mean (zero harmonic), sine, and cosine coefficients of the first harmonic were extracted by Fourier analysis and backprojected. All pixels were then re-aligned along the long axis of the left ventricle (hence double-angulation). Four defined projections (those chosen for angiography and echocardiography) were calculated. Mean, sine, and cosine coefficients were added (integrated) to form thick slices that contained the complete ventricle under investigation. The integration was performed for each of the four chosen views (fig 1). This view dependent addition of voxels means that the images represent a projection of the corresponding view rather than a single slice. Finally, we calculated the phase values from each pixel (fig 2). In summary, these procedures result in planar scans of the heart in three projections. In this way, the contraction patterns and initial inward motion of each cardiac chamber was assessed separately without significant overlap of other cardiac structures. ${ }^{26}$

We performed conventional radionuclide ventriculography to calculate the ejection fraction after each SPECT investigation (see table 1). Two investigators who were unaware of the results of the catheter mapping procedure independently evaluated the frames of double-angulated integrated SPECT.

\section{LOCALISATION OF ACCESSORY PATHWAYS BY}

\section{DA-ISPECT}

Figure 3 shows the eight different areas along the atrioventricular ring that were used to localise the accessory pathways. The results were compared with those from catheter mapping. When the sites were identical the correlation was 2 , when the sites were adjacent the correlation was 1 and in all other cases the correlation was 0 .

\section{LOCALISATION OF THE ORIGIN OF VENTRICULAR TACHYCARDIAS BY \\ DA-ISPECT}

We used a detailed diagram suggested by Kuchar $1989^{\circ}$ that divides the left ventricle into 24 different areas (fig 4) to localise the origin of the ventricular tachycardia within the left ventricle. The results from catheter mapping were compared with those from DA-ISPECT. We attempted to measure the distance between the origin of the tachycardias as determined by catheter mapping and
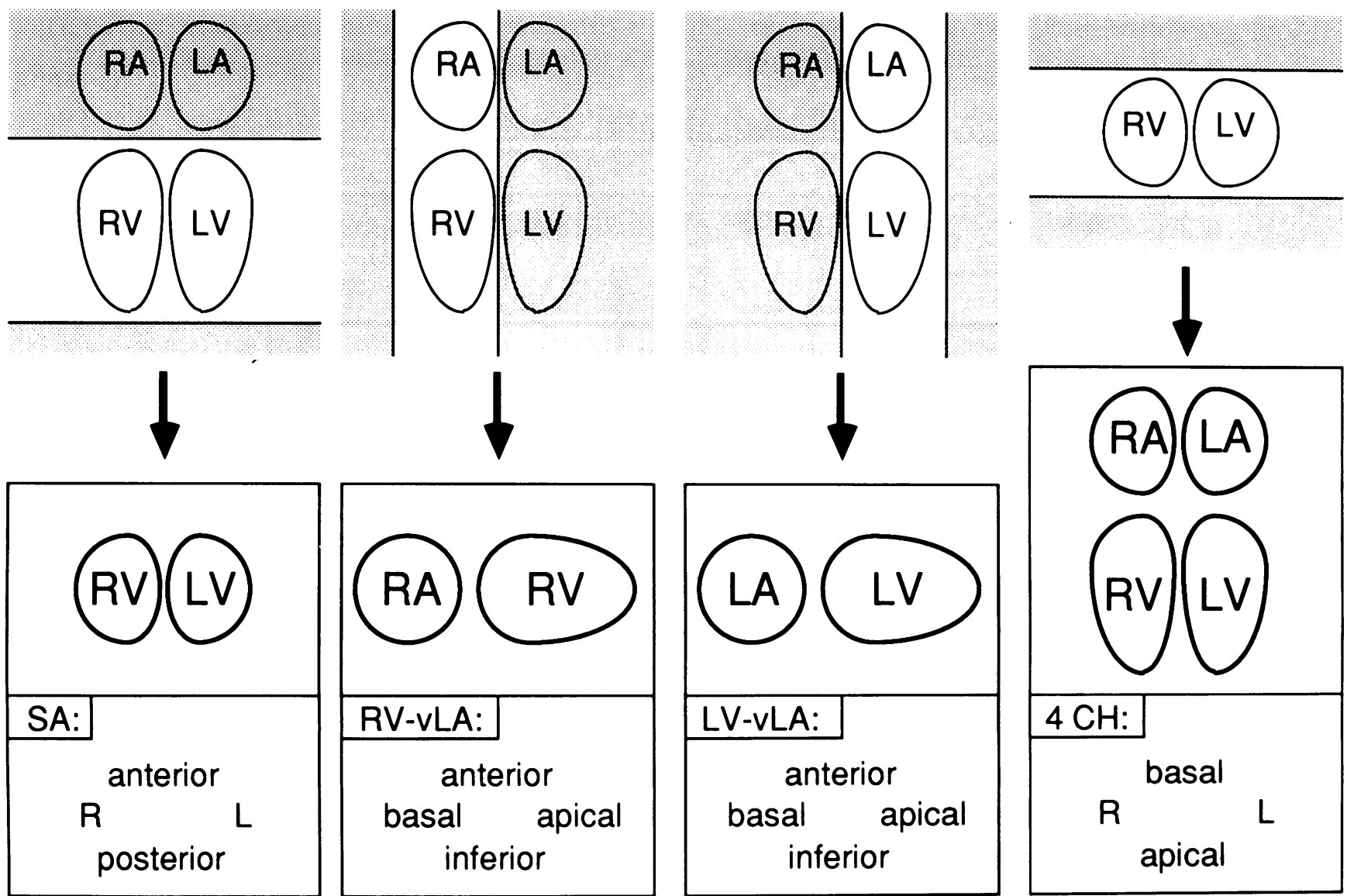

Figure 1 Double-angulated integrated SPECT (DA-ISPECT). Schematic display of the zones of integration (addition) after double angulation according to the long axis of the left ventricle. These zones are depicted non-darkened in the upper part of the figure and are used to calculate the "thick according to the long axis of the left ventricle. These zones are depicted non-darkened in the upper part of the figure and are used to calculate the "thick (LV-vLA), and four chamber view (4CH). 
Figure 2 Fourier phase images from $D A-I S P E C T$. The projections are arranged as in fig $1 . A$ colour code is shown on the right hand side. In the phase image the purple encodes the initial contraction and red the late contraction. The upper panel shows the contraction pattern in a healthy individual. There is early contraction (blue) at the septum (circle in the short axis view) and at the right free wall (circle in the long axis view of the right ventricle and four chamber view). The lower panel jattern in a WPW patient with an accessory pathway located anteroseptally (circle in short axis view and four chamber view).

For the surface

electrocardiogram see fig 8. shows the contraction
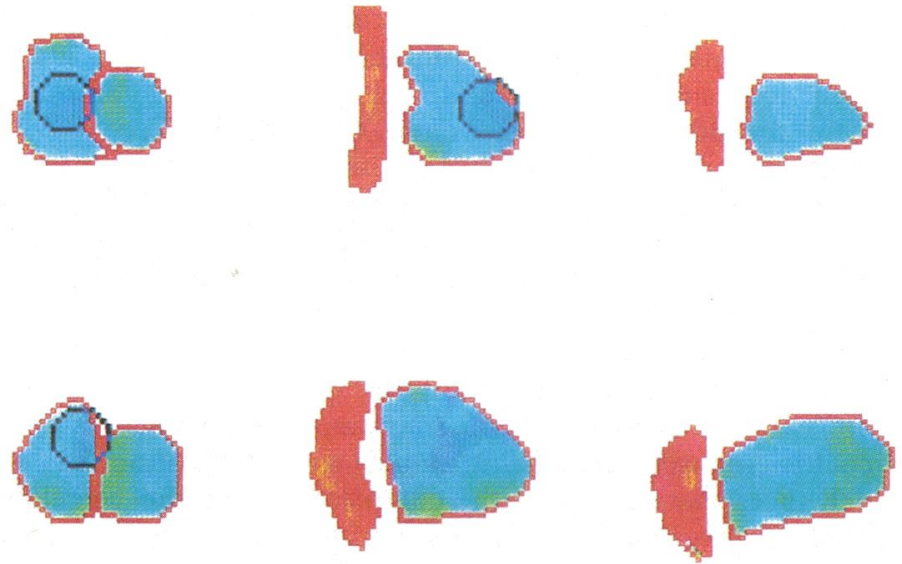

\section{R-long axis}

by DA-ISPECT.

All patients had given informed consent. The study complies with the declaration of Helsinki.

\section{Results}

CONTRACTION PATTERNS IN THE CONTROL GROUP

Sixteen (40\%) of the 40 patients did not have a recognisable contraction pattern that reflected normal physiology. In 24 patients (60\%) typical contraction patterns were seen. In five patients the initial contraction was seen at the anterior part of the right ventricle close to the apex (upper panel in fig 2). In

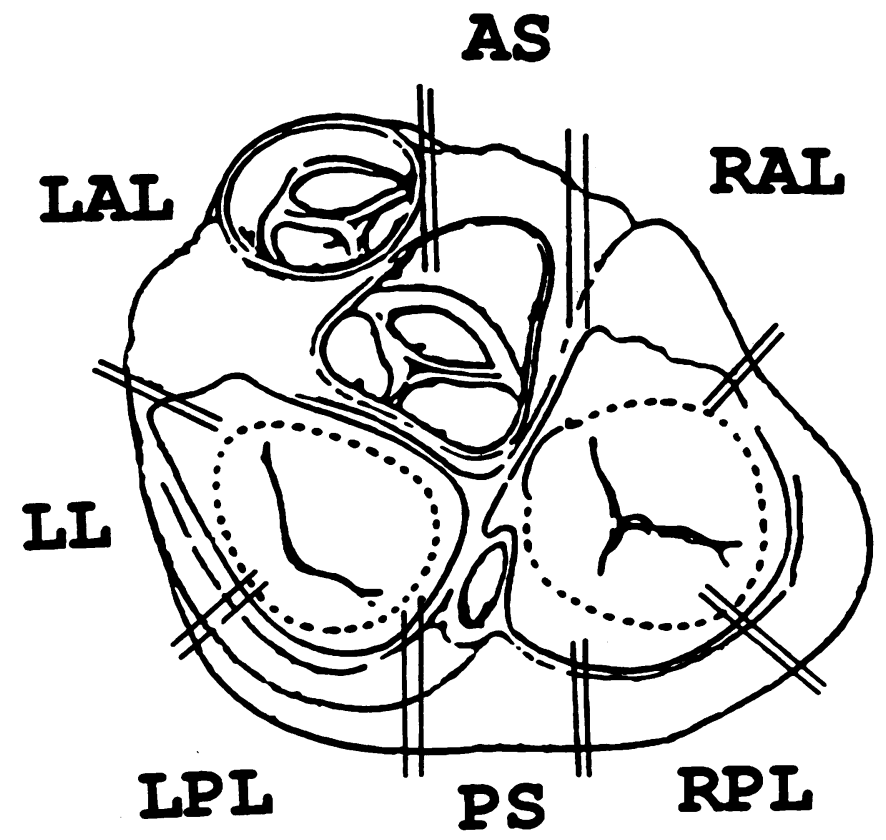

RI

Figure 3 Diagram of the atrioventricular ring for the evaluation of the site of the accessory pathway. There are eight areas: $A S$, anteroseptal; $R A L$, right anterolateral; $R L$, right lateral; $R P L$, right posterolateral; $P S$, posteroseptal; $L P L$, left posterolateral; $L L$, left lateral; $L A L$, left anterolateral.
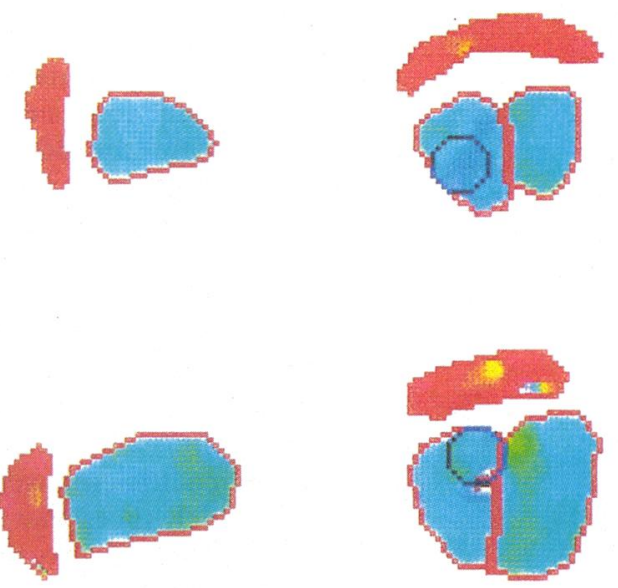

four other patients the initial contraction was detected paraseptally at the right and left base of the ventricles. The remaining 15 patients showed a combination of these two patterns. This accords with the electrophysiological findings of electrical excitation during sinus rhythm. ${ }^{27}$ The early contraction in the right ventricle may be explained by a rapid propagation of the electrophysiological impulse through the moderator band.

\section{CONTRACTION PATTERNS IN PATIENTS WITH} ACCESSORY PATHWAYS

Table 2 shows the results in the WPW patients. Additionally, Kent bundle localisation from surface electrocardiogram was assessed by the algorithms of Milstein et al ${ }^{6}$ and Gallagher et al. ${ }^{5}$

Figure 5 shows the DA-ISPECT image for a patient (case 22) with a left lateral accessory pathway confirmed by catheter mapping. Because of a long effective refractory period, atrioventricular conduction along the accessory pathway could be blocked by intravenous ajmaline $(50 \mathrm{mg})$, and a normal contraction pattern ensued (fig 5). Figure 6 shows the results of a DA-ISPECT study from a patient in whom catheter mapping identified a left posterolateral accessory pathway $3 \mathrm{~cm}$ distal to the ostium of the coronary sinus.

Four of the 15 patients had two accessory pathways. In one patient (case 17) no conduction could be detected from the ventricles to the atria through the accessory pathways. This patient had recurrent atrial fibrillation, resulting in rapid ventricular excitation through the bypass tract with rates of $250 / \mathrm{min}$. The preoperative DA-ISPECT study did not show a distinct and consistent early contraction site (fig 7). During preoperative catheter mapping one accessory pathway was found posteroseptally $2 \mathrm{~cm}$ distal to the coronary sinus with an effective refractory period of $200 \mathrm{~ms}$. During intraoperative mapping the atrial and ventricular insertion of the 

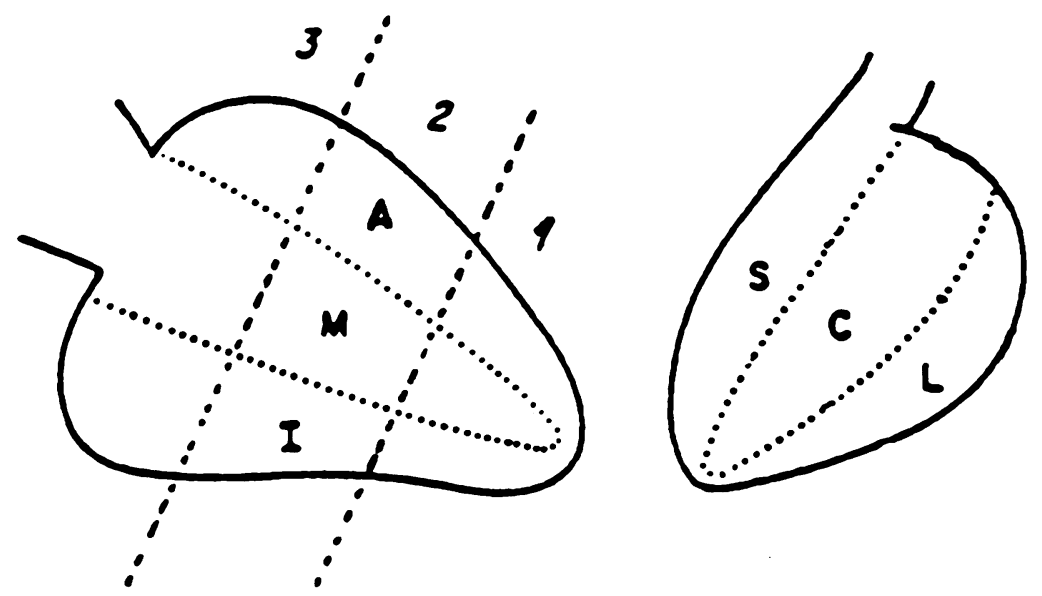

Figure 4 Grid showing 24 different areas of the left ventricle defined for the evaluation of the site of origin of ventricular tachycardias from surface electrocardiogram, catheter mapping, and DA-ISPECT. The left side of the figure shows the right anterior oblique $30^{\circ}$ projection and the right side shows the left anterior oblique $60^{\circ}$.projection. $1,2,3=$ apical, mid-ventricular, basal; $A$, anterior; $M$, middle; $I$, inferior; $S$, septal; $C$, central;

$I$, inferior. Adapted from Kuchar et al. ${ }^{8}$

accessory pathway was determined posteroseptally $1 \mathrm{~cm}$ anterior to the crux cordis. No further bundle was found. After operation intermittent atrioventricular conduction was seen with wide QRS complexes. The surface electrocardiogram indicated a second accessory pathway in the right lateral atrioventricular groove. ${ }^{4}$ The second DA-ISPECT study showed an initial contraction right posterolaterally during extensive pre-excitation (fig 7). The postoperative electrophysiological study showed a second accessory pathway at this site with an effective refractory period of $250 \mathrm{~ms}$. However, this bundle was operating only intermittently, which is why it was not found during the preoperative and intraoperative electrophysiological examination. When the preoperative DA-ISPECT study was retrospectively re-evaluated two sites of early contraction caused by two accessory pathways became apparent (fig 7).

One patient with WPW syndrome (case 16) had atrioventricular reentrant tachycar- dias $(260 / \mathrm{min})$. Figure 8 shows the surface electrocardiogram. The surface electrocardiogram did not show a distinct site of an accessory pathway. ${ }^{56}$ The electrophysiological study showed an accessory pathway with a very short effective refractory period $(180 \mathrm{~ms})$. Localisation of the bypass tract was not possible because repeated mechanical induction of atrial fibrillation by the mapping catheter resulted in ventricular rates of $250 / \mathrm{min}$. On one occasion induction of an atrioventricular reentrant tachycardia produced a left bundle branch block configuration with the same rate of $260 / \mathrm{min}$, indicating an accessory pathway located on the right side or within the septum..$^{28} \mathrm{~A}$ surface electrocardiogram recorded during maximum preexcitation (rapid atrial stimulation) indicated a right lateral accessory pathway. ${ }^{6}$ The DAISPECT study showed clear evidence of early anteroseptal contraction (fig 2). Later, after oral administration of flecainide ( $300 \mathrm{mg}$ ) day), successful radiofrequency catheter ablation confirmed the presence of an accessory pathway located $1 \mathrm{~cm}$ anterior to the bundle of His. In the DA-ISPECT study after catheter ablation without preexcitation, the beginning of the contraction could be localised posteriorly only-a small distance from the site detected by the first DAISPECT study. In patient 19, another successful radiofrequency catheter ablation ${ }^{23}$ confirmed the exact atrial insertion site of the accessory pathway. In patient 12 with two bypass tracts, the site of the second pathway was localised from DA-ISPECT only by retrospective re-evaluation.

Table 2 summarises the results. In 10 of the 14 patients in whom catheter mapping was performed the DA-ISPECT data correlated well with the catheter mapping data (fig 3). In three patients DA-ISPECT showed an area of early contraction next to the area found by catheter mapping. In one patient no correlation could be found between the two methods. In the four patients with two accessory bundles, only one pathway could be

Table 2 Localisation of initial excitation and contraction in patients with Wolff-Parkinson-White syndrome

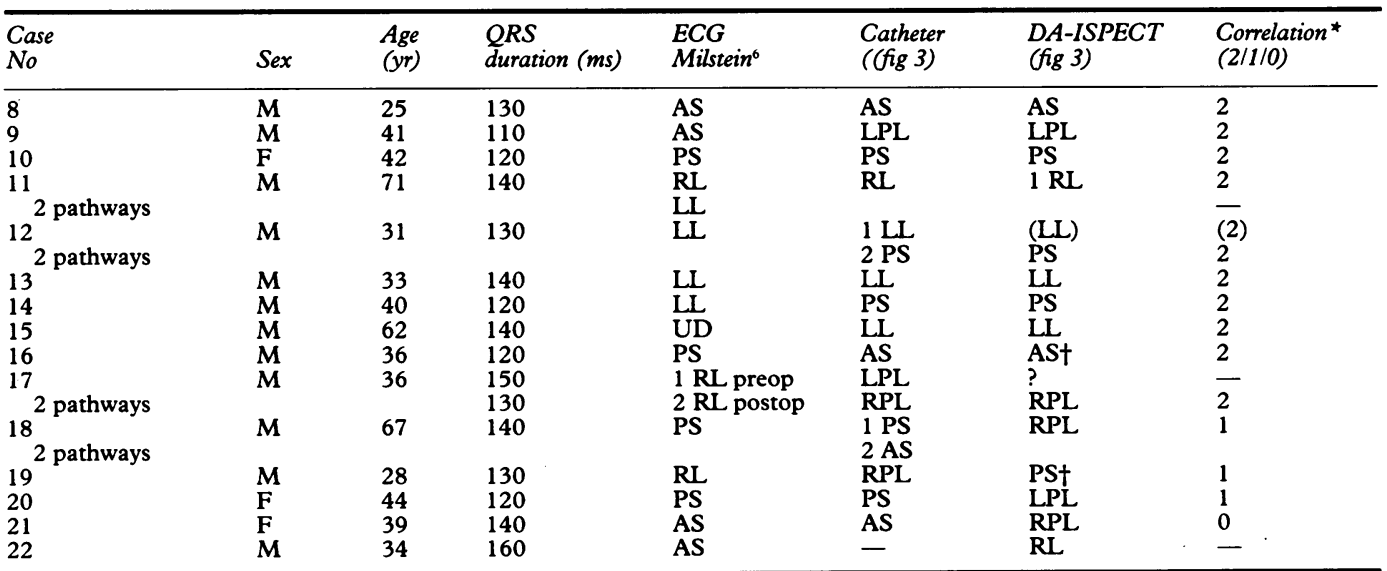

Site of the accessory pathway determined from surface electrocardiogram according to Milstein et al ${ }^{\circ}:$ AS, anteroseptal; LL, left lateral; RL right lateral; PS, posteroseptal; UD, undetermined. See fig 3 for designation of the site of the accessory pathways lateral; RL, right lateral; PS, posteroseptal; UD, undete

determined from catheter mapping and DA-ISPECT.
*Correlation: 2 , same area detected by catheter mapping and DA-ISPECT; 1 , area detected by catheter mapping was adjacent to *Correlation: 2 , same area detected by catheter mapping and DA-ISPECT; 1 , area detected by catheter
the area detected by DA-ISPECT; 0 , different areas detected by catheter mapping and DA-ISPECT. †Successful radiofrequency catheter ablation. 
nuclear redicine, UIn
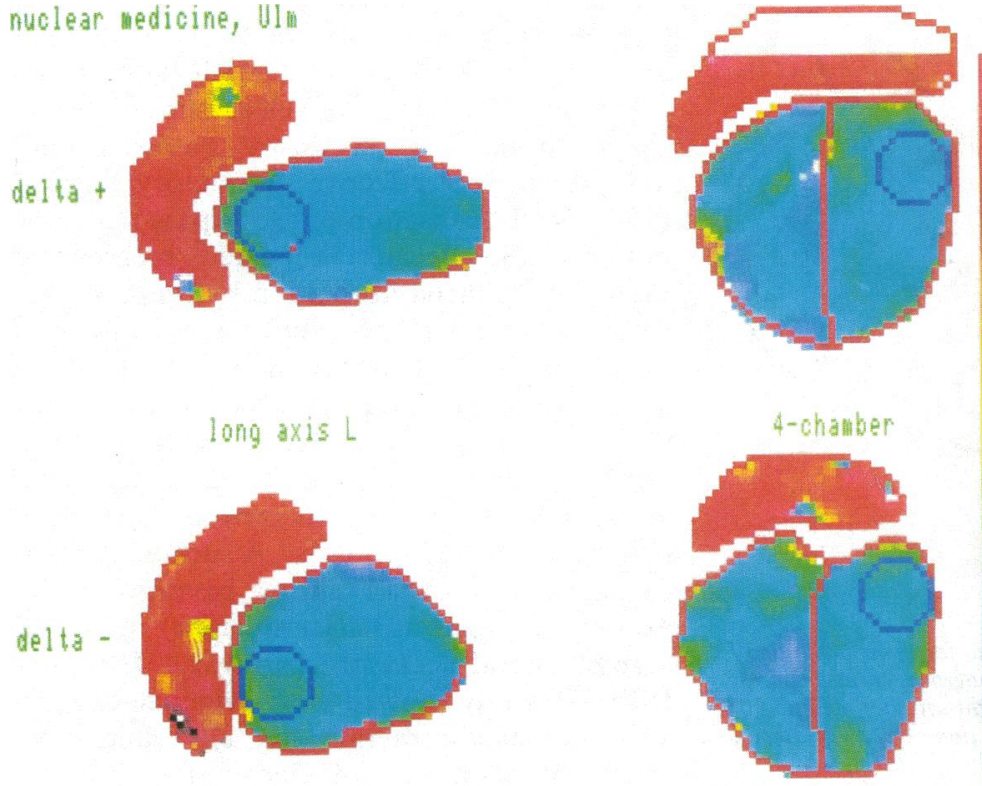

Figure 5 Fourier phase images from DA-ISPECT of a patient (case 4) with a left lateral accessory pathway. The upper panel of the figure shows the long axis view of the left ventricle and the four chamber view during anterograde conduction along the accessory pathway. The lower panel show the same views after administration of $50 \mathrm{mg}$ ajmaline, which led to normal conduction. The circle indicates early contraction in the presence of the delta wave in contrast to the contraction pattern during normal conduction.
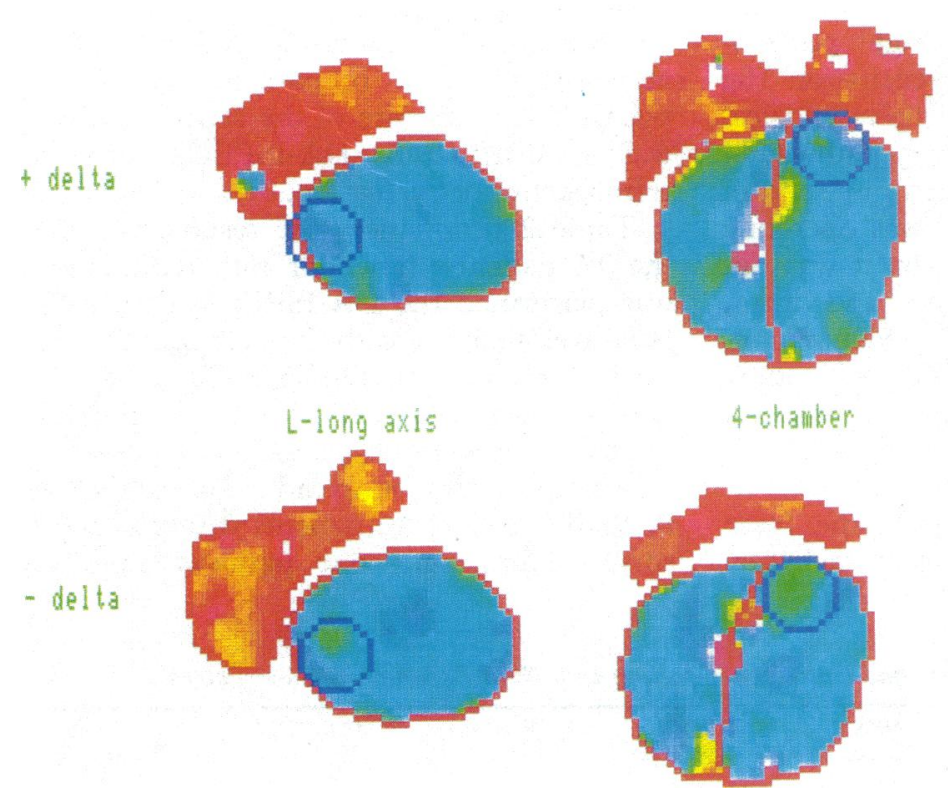

Figure 6 Fourier phase images from DA-ISPECT of a patient (case 9) with a left posterolateral accessory pathway found $3 \mathrm{~cm}$ distal from the ostium of the coronary sinus during catheter mapping. The figure is arranged like fig 5.

detected by DA-ISPECT. Localisation of accessory pathways from standard 12 lead surface electrocardiogram according to the criteria proposed by Gallagher et $a l^{5}$ and Milstein et $a l^{6}$ was less accurate than DAISPECT.

In two of the three patients with a bypass tract in the septum (cases 16, 20 and 21), DA-ISPECT was not able to detect the location of the accessory pathway.
CONTRACTION PATTERNS IN THE PATIENTS

WITH VENTRICULAR TACHYCARDIAS

Table 3 shows the results in the patient group studied by DA-ISPECT during ventricular tachycardia. In two patients (cases 2 and 7) catheter ablation was not completely successful, though subsequent induction of the ventricular tachycardia was more difficult. The patients have not had tachycardia for 12 and 13 months respectively.

Figure 9 shows the results of a DAISPECT study in patient 2 , who had an anterior wall aneurysm. The contraction patterns from phase analysis during ventricular tachycardia and during sinus rhythm were completely different. During ventricular tachycardia the contraction started in the mid-ventricle at the anterior part of the septum. This coincided with the origin of the tachycardia found by catheter mapping and was confirmed by catheter ablation. Figure 10 shows a DA-ISPECT study from a patient (case 6) with a posterior wall aneurysm and an ejection fraction of $33 \%$ during ventricular tachycardia. The initial contraction was located at the lateral side of the base of the left ventricle. Catheter mapping showed the site of origin of the ventricular tachycardia with a diastolic potential $100 \mathrm{~ms}$ before the QRS complex at the base of the ventricle, but this lay posteriorly, about $4 \mathrm{~cm}$ from the site of the initial contraction. In another patient (case 7) with a large anterior wall aneurysm (ejection fraction during ventricular tachycardia $17 \%$ ) the site of origin of the ventricular tachycardia determined by catheter mapping was about $5 \mathrm{~cm}$ from the site detected by DA-ISPECT (table 3). The interpretation of the DA-ISPECT image was difficult because large areas showed early contraction in this patient. This was also true of patient 4 , who also showed poor left ventricular performance. A second early contracting area (fig 4) was identified from the DA-ISPECT image.

In five of the patients the site of initial contraction accorded with the origin of the ventricular tachycardia (table 3). The differences seen in two of the patients will be discussed. The mean difference between the localisation of the focus by catheter mapping and by DAISPECT was $2.4 \mathrm{~cm}$ (range $1-5 \mathrm{~cm}$ ).

\section{Discussion}

These are the first clinical experiences to be reported with the new method of doubleangulated integrated SPECT. This new method of evaluating SPECT data has the advantage of showing ventricles in any chosen view. Difficulties, limitations, and possible clinical applications will be discussed for each patient group.

\section{CONTRACTION PATTERN IN THE CONTROL}

\section{GROUP}

Only $60 \%$ of the control patients had a typical contraction pattern. This pattern of contraction corresponds to physiological excitation of ventricular myocardium via the 
Figure 7 Fourier phase images from DA-ISPECT of a patient (case 17) with two accessory pathways. The upper set of the preoperative phase images shows no consistent site of early contraction (early contraction marked by circles at two different places-left posterolateral and right posterolateral). In the lower part of the figure the phase image clearly shows initial contraction in the right posterolateral area (indicated by the circle), which can now also be identified in the preoperative image (see short axis view and long axis view of the right chamber). The phase images are arranged as in fig 1 .

Figure 8 Twelve lead surface electrocardiogram from a WPW patient (case 16), whose accessory pathway initially could not be localised by invasive means because of repeated mechanically induced atrial fibrillation. Extr, limb leads.
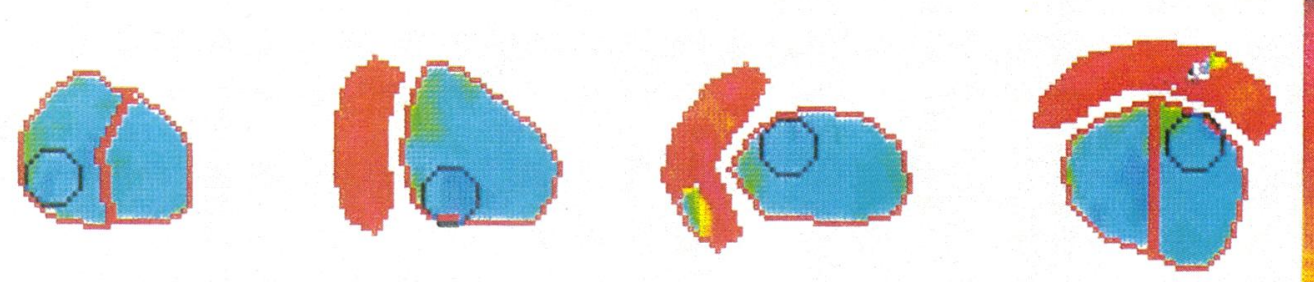
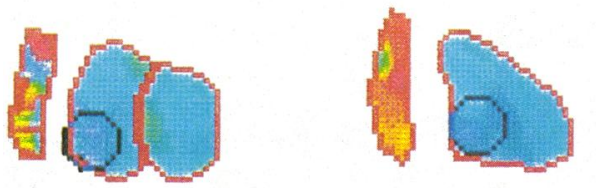

short axis

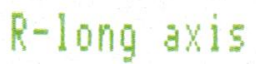

His-Purkinje system, as reported for the human heart by Durrer et al. ${ }^{27}$ In the remaining control patients no distinct contraction pattern was identified. Even in 13 healthy dogs previously studied by DA-ISPECT, two animals showed contraction patterns that were different from physiological expectations. The contraction patterns in healthy individuals therefore seem to vary probably because of interindividual temporal variation in the electrical excitation of ventricular
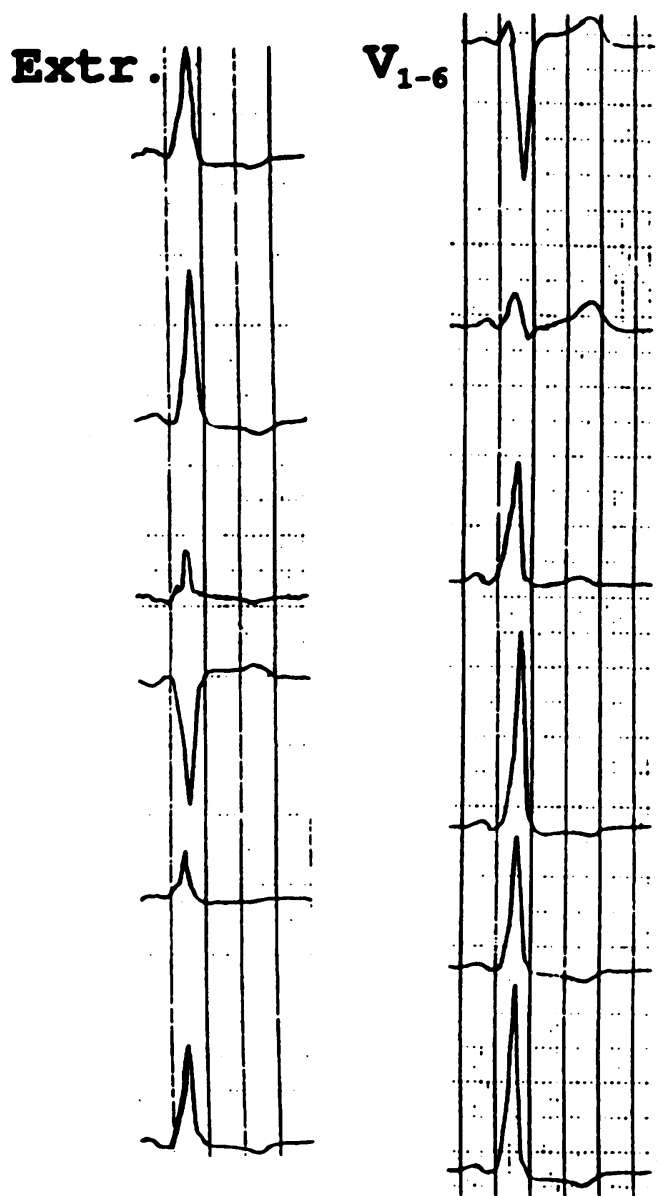

myocardium. This makes it more difficult to evaluate the results from the DA-ISPECT in WPW patients.

CONTRACTION PATTERNS IN WPW SYNDROME

Because there is no standard contraction pattern in controls, the investigator performing DA-ISPECT needs to know whether the patient being studied has a delta wave. In the clinical setting this information is always available. Each beat in a WPW patient with a delta wave is a combination of normal excitation along the His-Purkinje system and of excitation along the accessory pathway. The propagation of the earliest electrical excitation and ensuing contraction from the ventricular insertion site of the accessory pathway is slower than the electrical excitation along the physiological route (His-Purkinje system) which begins later. This makes it difficult to analyse the contraction patterns of such fusion beats. In seven of the patients the normal contraction pattern was seen when conduction through the accessory pathway was blocked (figs 5 and 6). This facilitated the analysis. In the WPW patients preexcitation was not increased by either pacing the atria or by drugs to broaden the delta wave. Pacing is an invasive procedure. Nevertheless, the effect of atrial pacing should be investigated to assess the diagnostic potential of DAISPECT in WPW patients paced from the atria.

\section{CONTRACTION PATTERNS IN VENTRICULAR}

TACHYCARDIA

Compared with WPW patients preexcitation was always present during ventricular tachycardia and ventricular pacing (which was performed in two patients). This facilitates the evaluation of the DA-ISPECT results in the patients with ventricular tachycardia. We found a good correlation between the results of DA-ISPECT and catheter mapping regarding the site of origin of the ventricular tachycardia (table 3).

During the procedure of catheter mapping the pathway of slow conduction is localised 
Table 3 Correlations between surface electrocardiogram, catheter mapping, and DAISPECT during ventricular tachycardia

\begin{tabular}{llllll}
\hline Case & ECG & DA-ISPECT & $\begin{array}{l}\text { Catheter } \\
\text { mapping }\end{array}$ & Correlation $^{*}$ & $\begin{array}{l}\text { Spatial } \\
\text { difference (cm) } \dagger\end{array}$ \\
\hline 1 & $2 / \mathrm{I} / \mathrm{S}$ & 3/I/S & $3 / \mathrm{I} / \mathrm{S}$ & 2 & 1 \\
2 & $2 / \mathrm{A} / \mathrm{S}$ & $2 / \mathrm{A} / \mathrm{S}$ & $2 / \mathrm{A} / \mathrm{S}$ & 2 & 1 \\
3 & $2 / \mathrm{S}$ & $2 / \mathrm{I} / \mathrm{S}$ & $2 / \mathrm{S}$ & 2 & 1 \\
4 & $2 / \mathrm{M} / \mathrm{S}$ & $2 / \mathrm{M} / \mathrm{S}$ & $2 / \mathrm{M} / \mathrm{S}$ & 2 & 2 \\
5 & $2 / \mathrm{S}$ & $2 / \mathrm{S}$ & $2 / \mathrm{S}$ & 2 & 3 \\
6 & $2 / \mathrm{L}$ & $2 / \mathrm{M} / \mathrm{L}$ & $2 / \mathrm{C}$ & 0 & 4 \\
7 & $2 / \mathrm{A} / \mathrm{L}$ & $2 / \mathrm{M} / \mathrm{L}$ & $3 / \mathrm{M} / \mathrm{S}$ & 0 & 5 \\
\hline
\end{tabular}

The abbreviations for area designation are given in fig 4 .

*Correlation: 2, same area detected by catheter mapping and DA-ISPECT; 1 , area detected by catheter mapping in directly adjacent to the area detected by DA-ISPECT; 0, different areas detected by catheter mapping and DA-ISPECT.

†Spatial difference between sites determined by catheter mapping and by DA-ISPECT.

Figure 9 Phase images from $D A$-ISPECT of a patient with ventricular tachycardia (case 2). The upper row shows the contraction pattern during sinus rhythm. There is slight contraction in the apex of the left ventricle (anterior wall aneurysm). The lower panel shows phase images during ventricular tachycardia.

The contraction started septally in the anterior wall of the mid-ventricular area (see fig 4), as indicated by the circle. The projections are arranged as in fig 1 .

by recording diastolic potentials and by other criteria. ${ }^{4}$ This small amount of electrophysiological activity in severely damaged myocardium does not lead to contraction. It is assumed that DA-ISPECT identifies healthy-that is-contractile myocardium that shows mechanical activity at the start of contraction. This may correspond to the site at the end of the slow conducting pathway ${ }^{29}$ a fact that may explain the difference between catheter mapping and DA-ISPECT results in patient 6. At present only invasive means such as catheter mapping (feasible in $16 \%)^{30}$ or mapping during surgery ${ }^{31}$ are able to detect the complete pathway of slow conduction.

In patient 4 , who had an ejection fraction of only $17 \%$ during tachycardia, large areas of early contraction were identified. These may reflect areas of reduced contractility and could be the cause of the poor result of DAISPECT in this case.

Animal studies are needed to clarify the specificity of DA-ISPECT for identifying sites of excitation and subsequent contraction.

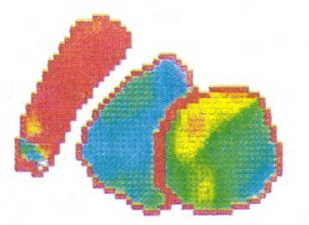

\section{ADVANTAGES AND DISADVANTAGES OF} DA-ISPECT

Advantages of the DA-ISPECT in WPW patients

- The results indicate that DA-ISPECT is more exact and reliable than the surface electrocardiogram in localising accessory pathways.

- It is non-invasive and though subsequent invasive procedures cannot be replaced by DA-ISPECT they are probably shortened by reducing the time required for fluoroscopy.

- Unlike DA-ISPECT catheter mapping usually identifies the atrial insertion of the accessory bundle. For catheter ablation of left-sided accessory pathways it is imperative to localise the ventricular insertion site because the atrial and ventricular insertions of the bypass tract may differ. ${ }^{32}$
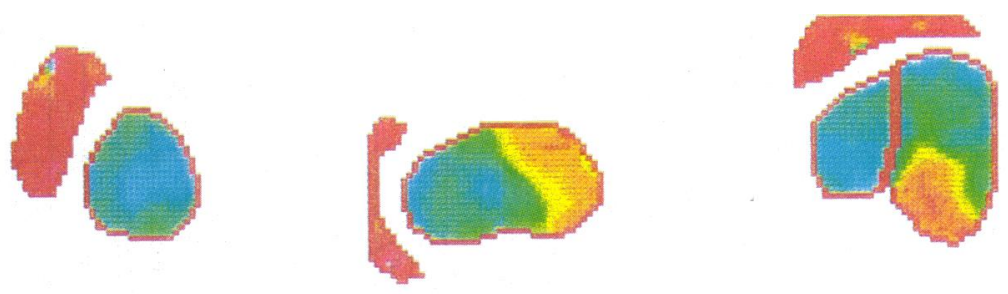

those rare patients in whom catheter mapping is not suitable.

- With DA-ISPECT there is only a little radiation exposure from the radionuclide.

Advantages of DA-ISPECT in patients with ventricular tachycardia

- DA-ISPECT takes less time than catheter mapping, it does not need an arterial puncture, and it is better tolerated by the patients.

- The resolution of the site of origin of the ventricular tachycardia is good enough for preoperative evaluation.

- Catheter mapping is contraindicated when a left ventricular thrombus is present.

\section{Disadvantages of DA-ISPECT in WPW patients}

- The success of DA-ISPECT is limited when the preexcitation is small (narrow delta wave).
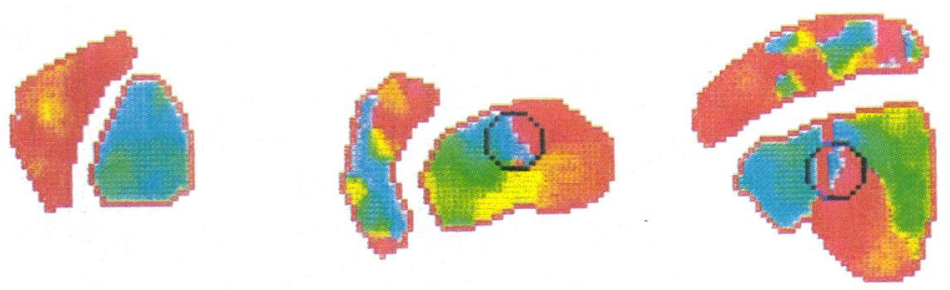
Figure 10 Phase images from DA-ISPECT of a patient with an inferior aneurysm (case 6) during sinus rhythm (upper row) and during ventricular tachycardia (lower row). In contrast to the contraction pattern during sinus rhythm, the initial contraction during the ventricular tachycardia is located in the middle of the left ventricle, laterally and in the mid-ventricular area (see fig 3), as indicated by the circle.
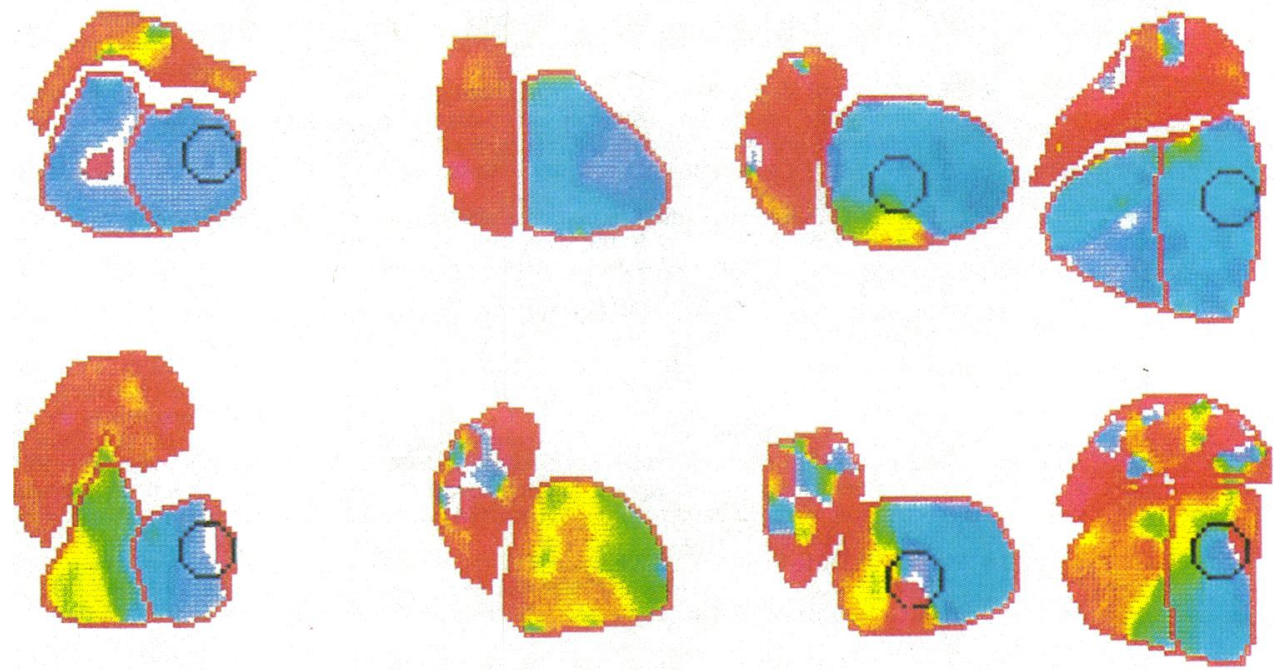

R-long axis
In subsequent investigations preexcitation could be increased by pacing or drugs to improve the results of DA-ISPECT.

- The presence and location of multiple accessory pathways cannot be detected.

- Concealed bypass tracts are not detected..$^{33}$

- The functional properties of. accessory pathways cannot be evaluated.

Disadvantages of DA-ISPECT in patients with ventricular tachycardias

- For data acquisition tachycardia must be induced by a stimulation catheter.

- The tachycardia must be tolerated for $20 \mathrm{~min}$. This limits the number of patients in whom this method is suitable. Attempts to shorten the total time of data acquisition are in progress. The tachycardia rate can be reduced by drugs. This results in a better haemodynamic tolerance, as it does in patients who require catheter ablation.

- DA-ISPECT relies on mechanical contraction for the evaluation of excitation. But electrical excitation, which is recorded by catheter mapping, does not always lead to contraction.

- The success of DA-ISPECT is limited in patients with poor left ventricular performance.

\section{Conclusions}

Catheter mapping and intraoperative mapping remain the standard methods of localising accessory pathways and the origin of ventricular tachycardias. The results of DAISPECT are compared with the results of these methods. But in catheter mapping and even intraoperative mapping spatial discrimination is limited. ${ }^{1}$ The most exact method of localisation seems to be successful radiofrequency ablation, ${ }^{23}$ because it destroys small areas. ${ }^{24}$

DA-ISPECT is a new method of evaluating cardiac blood pool SPECT. It has limitations and disadvantages in the evalua- tion of patients with arrhythmias. Nevertheless it is an additional tool to assess these patients before invasive procedures. In a few patients DA-ISPECT provides additional information that influences treatment (for example, in patients in whom atrial fibrillation is easily mechanically induced and in patients with thrombi). To date only a few patients have been investigated by DAISPECT. As the software becomes available to other centres, the method could be evaluated in more patients and its clinical usefulness could be determined.

1 Josephson ME, Harken AH, Horowitz LN. Endocardial excision. A new surgical technique for treatment of recurrent ventricular tachycardia. Circulation 1979;60: 1430-9.

2 Evans GT, Scheinmann MM. Catheter ablation for control of ventricular tachycardia: A report of the percutaneous cardiac mapping and ablation registry. $P A C E$ 1986;9:1391-4.

3 Gallagher JJ. Localization of accessory atrioventricular pathways: what's the "gold standard"? PACE 1987; 10:583-4.

4 Josephson ME, Horowitz LN, Waxman HL, Spielman SR, Untereker WJ, Marchlinksi FE. Role of catheter mapping in evaluation of ventricular tachycardia. In: Josephson ME, ed. Ventricular tachycardia. Mechanisms and management, Mount Kisco, NY: Futura Publishing Company, Inc, 1982;309-30.

5 Gallagher JJ, Prittchett ELC, Sealy WC, Kasell J, Wallace AG. The preexcitation syndromes. Prog Cardiovasc Dis 1978;20:285-327.

6 Milstein S, Sharma AD, Guiraudon GM, Klein GJ. An algorithm for the electrocardiographic localization of accessory pathways in the Wolft
drome. PACE 1987;10:555-63.

7 Josephson ME, Horowitz LN, Waxman HL, et al. Sustained ventricular tachycardia of the 12-lead electrocardiogram in localizing site of origin. Circulation 1981; 64:257-72.

8 Kuchar DL, Ruskin JN, Garan H. Electrocardiographic localization of the site of origin of ventricular tachycardia in patients with prior myocardial infarction. $\mathcal{f} \mathrm{Am}$ Coll Cardiol 1989;13:893-900.

9 Geffers H, Adam WE, Bitter F, Sigel H, Stauch M Radionuclide ventriculography I. Fundamentals and methods. Nucl Med 1978;17:206-10.

10 Adam WE, Tarkowska A, Bitter F, Stauch M, Geffers H. Equilibrium (gated) radionuclide ventriculography. Cardiovasc Radiol 1979;2:161-73.

11 Cardiovasc Radiol 19 , Radionuclide ventriculography equilibrium gated blood pool scanning - its present clinical position and recent developments. Eur f Nucl Med 1988;13:637-47.

12 Botvinick EH, Frais MA, Shosa DW, et al. An accurate means of detecting and characterizing abnormal patterns of ventricular activation by phase image analysis. Am $\mathcal{F}$ Cardiol 1982;50:289-98. 
13 Pavel DG, Byrom E, Lam W, Mayer-Pavel C, Swyrin S, Pietras R. Detection and quantification of regional wall motion abnormalities using phase analysis of equilibrium gated cardiac studies. Clin Nucl Med 1983;8. 315-21

14 Chan W, Kalff V, Mach Donald D, et al. Topography of preemptying ventricular segments in patients with Wreemptying ventricular segments in patients with phase mapping and esophageal pacing. Circulation 1983; 67:1139-46.

15 Nakajima K, Bunko H, Tada A, et al. Phase analysis in the Wolff-Parkinson-White syndrome with surgical proven accessory conduction pathways: Concise communication. 7 Nucl Med 1984;25:7-13.

16 Dormehl I, Bitter F, Henze E, Adam WE, Weismüller P. An evaluation of the diagnostic efficacy of phase analysis of data from radionuclide ventriculograms in patients with Wolff-Parkinson-White syndrome. Eur $7 \mathrm{Nucl} \mathrm{Med}$ 1985;11:150-5.

17 Swiryn S, Pavel D, Byrom E, et al. Sequential regiona phase mapping of radionuclide gated biventriculograms in patients with sustained ventricular tachycardia: Close correlation with electrophysiologic characteristics. $\mathrm{Am}$ correlation with electrophys

18 McCarthy DM, Makler PT, Waxman HL, et al. Fourier phase image during ventricular tachycardia: Relationship to endocardial site of origin [abstr]. $\mathcal{f} \mathrm{Am}$ Coll Cardiol 1983;1:712.

19 Le Guludec D, Sebag C, Bourguignon M, et al. Regional phase mapping of radionuclide gated biventriculograms in patients with sustained ventricular tachycardia. In: Aliot E, Lazarra R, eds. Ventricular tachycardias-from mechanism to therapy. Dordrecht/Boston Lancaster: Martinus Nijhoff Publishers, 1987;231-45.

20 Weismüller P, Henze E, Adam WE, Roth J, Bitter F, Stauch M. Parametric imaging of experimentally simulated Wolff-Parkinson-White syndrome conduction lated Wolff-Parkinson-White syndrome conduction
abnormalities in dogs. Am f Physiol Imaging 1986;1: abnorma $208-13$.

21 Weismüller P, Clausen M, Henze E, et al. Localization of premature and of ectopic ventricular depolarization by means of a new tomographic radionuclide technique. $Z$ Kardiol 1990;79:529-34.

22 Clausen $\mathrm{M}$, Henze E, Weller $\mathrm{R}$, et al. Tomographic radionuclide ventriculography ( $\mathrm{RNV}$ ) with reconstruc- ted planar views perpendicular to the body axis (ISPECT). Nucl Compact 1990;21:95-9.

23 Jackmann WM, Wang X, Friday K, et al. Catheter ablation of accessory atrioventricular pathways (WolffParkinson-White syndrome) by radiofrequency current. N Engl f Med 1991;324:1605-11.

24 Borggrefe M, Hindricks G, Haverkamp W, Budde Th, Breithardt G. Radiofrequency ablation. In: Zipes DP, Jalife J, eds. Cardiac Electrophysiology. From cell to Jalife J, eds. Cardiac Electrophysiology. From cell to

25 Morady F, Scheinman MM, Di Carlo LA, et al. Catheter ablation of ventricular tachycardia with intracardiac shocks: results in 33 patients. Circulation 1987;75 $1037-49$.

26 Clausen $\dot{M}$, Weismüller P, Weller R, et al. Doubleangulated, integral emission-computed tomograph (DA-ISPECT) in radionuclide ventriculography (RNV) with reconstructed planar views oriented according to the long axis of the heart. Nucl Compact 1990;21:167-70.

27 Durrer D, van Dam RT, Freud GE, Janse MJ, Meijler FL, Arzbaecher RC. Total excitation of the isolated human heart. Circulation 1970;41:899-912.

28 Coumel P, Attuel P. Reciprocating tachycardia in overt and latent preexcitation. Influence of functional bundle branch block on the heart rate of the tachycardia. Eur $\mathcal{F}$ Cardiol 1974;1:423-36.

29 El-Sherif N, Mehra R, Gough WB, Zeiler RH. Reentrant ventricular arrhythmias in the late myocardial infarction period. Circulation 1983;68:644-56.

30 Kuck K-H, Schlüter M, Geiger M, Siebels J. Vollständiges "Mapping" des Areals langsamer Leitun bei ventrikulären Tachykardien [abstr]. Z Kardiol 1991; 80:49.

31 Harris L, Downar E, Micklebourough L, Shaikh $\mathbf{N}$, Parson I. Activation sequence of ventricular tachycardia: endocardial and epicardial mapping studies in the human ventricle. $₹ \mathrm{Am}$ Coll Cardiol 1987;10:1040-7.

32 Jackman WM, Kuck KH, Friday KJ, Lazarra R. Catheter recordings of atrioventricular pathway activation. In: Zipes DP, Jalife J, eds. Cardiac electrophysiology. From Zipes DP, Jalife J, eds. Cardiac electrophysiology. From cell to bed

33 Neuss H, Schlepper M, Thormann J. Analysis of re-entry mechanisms in three patients with concealed WolffParkinson-White syndrome. Circulation 1975;51:75-81. 